\title{
Inventory Management for Deteriorating Items with Salvage Value under Time Varying Demand Condition
}

\author{
Srichandan Mishra ${ }^{1}$, S.P. Mishra ${ }^{2}$, N.Mishra ${ }^{3}$, J.Panda ${ }^{4,5}$, U.K.Misra ${ }^{6, *}$ \\ ${ }^{1}$ Department of Mathematics, Govt. Science College, Malkangiri, Odisha, India \\ ${ }^{2}$ Swarnamayee Nagar, Berhampur, Odisha, India \\ ${ }^{3}$ Department of MBA, Berhampur University, Berhampur, Odisha, India \\ ${ }^{4}$ Department of Commerce, Berhampur University \\ ${ }^{5}$ Department of Mathematics, Odisha, India \\ ${ }^{6}$ Department of Mathematics, N I S T, Berhampur, Odisha, India \\ *Corresponding author: umakanta_misra@yahoo.com
}

Received May 09, 2014; Revised May 20, 2014; Accepted May 21, 2014

\begin{abstract}
In this paper we discuss the development of an inventory model for deteriorating items which investigates an instantaneous replenishment model for the items under cost minimization. The salvage value is incorporated to the deteriorated units. The result is illustrated with numerical example.
\end{abstract}

Keywords: demand, optimal control, salvage value, inventory system

Cite This Article: Srichandan Mishra, S.P. Mishra, N.Mishra, J.Panda, and U.K.Misra, "Inventory Management for Deteriorating Items with Salvage Value under Time Varying Demand Condition.” American Journal of Applied Mathematics and Statistics, vol. 2, no. 3 (2014): 160-162. doi: 10.12691/ajams-2-3-11.

\section{Introduction}

The deterioration of item is a realistic phenomenon in any inventory system. It is reasonable to note that a product may be understood to have a lifetime which ends when utility reaches zero. The decrease or loss of utility due to decay is usually a function of the on-hand inventory. For items such as steel, hardware, glassware and toys, the rate of deterioration is so low that there is little need for considering deterioration in the determination of the economic lot size. But some items such as blood, fish, strawberry, alcohol, gasoline, radioactive chemical, medicine and food grains (i.e., paddy, wheat, potato, onion etc.) deteriorate remarkably overtime. In the history of inventory theory, the deteriorating inventory models have been continuously modified to become more practicable and realistic. It has been observed that failure of many items can be expressed in terms of Weibull distribution.

Ghare and Scharder [4] first formulated a mathematical model with a constant deterioration rate. Wee [9] developed EOQ models to allow deterioration and an exponential demand pattern. Emmone [3] established a replenishment model for radioactive nuclide generators. The assumption of the constant deterioration rate was relaxed by Covert and Philip [2], who used a twoparameter Weibull distribution to represent the distribution of time to deterioration. This model was further generalized by Philip [6] by taking three-parameter Weibull distribution for deterioration.

Variation in the demand rate plays an important role in the inventory management. Therefore, decisions of inventory are to be made because of the present and future demands. Demand may be constant, time-varying, stockdependent, price-dependent etc. The constant demand is valid, only when the phase of the product life cycle is matured and also for finite periods of time. Wagner and Whitin [8] discussed the discrete case of the dynamic version of EOQ. EOQ models for deteriorating items with trended demands were considered by Bahari-Kashani [1], Goswami and Chaudhuri [5]. R.P.Tripathi [7] developed a model under time-varying demand rate and holding cost.

In the present paper, an economic order quantity model is developed for Weibull deteriorating items for time varying demand rate. An attempt has been made to obtain optimal purchase quantity with some salvage value associated to the deteriorated units during the cycle time.

\section{Assumptions and Notations}

Following assumptions are made for the proposed model:

i. Time varying Demand rate is considered.

ii. Single inventory will be used.

iii. Lead time is zero.

iv. Shortages are not allowed.

$v$. Replenishment rate is infinite.

vi. Time horizon is finite.

vii. There is no repair of deteriorated items occurring during the cycle.

Following notations are made for the given model:

$I(t)=$ On hand inventory at time $t$.

$R(t)=\lambda t^{-p}=$ Time varying demand rate where $\lambda>0$.and $0<p<1$. 
The distribution of the time to deterioration of an item follows the Weibull distribution $\theta(t)$ where $\theta(t)=\alpha \beta t^{\beta-1}, \quad$ for $0 \leq t \leq T$, where $\alpha(0 \leq \alpha \leq 1)$ denotes the scale parameter, $\beta(\beta \geq 1)$ denotes the shape parameter and $t(t>0)$ is time for deterioration.

$I(0)=Q=$ Inventory at time $t=0$.

$T=$ Duration of a cycle.

$A=$ The ordering cost per order during the cycle time.

$p_{c}=$ The purchasing cost per unit item.

$d_{c}=$ The deterioration cost per unit item.

$h_{c}=$ The holding cost per unit item.

$\gamma p_{c}=$ The salvage value associated to the deteriorated units during the cycle time

where $0 \leq \gamma<1$.

\section{Formulation}

Let $I(t)$ be the on hand inventory at time $t \geq 0$ where $0 \leq t \leq T$. In the interval $[0, T]$ the stock will be decreased due to the effect of deterioration and demand. At time T, the inventory level reaches zero. At time $t+\Delta t$, the on hand inventory in the interval $[0, T]$ will be

$$
I(t+\Delta t)=I(t)-\theta(t) I(t) \cdot \Delta t-\lambda t^{-p} . \Delta t
$$

Dividing by $\Delta t$ and then taking as $\Delta t \rightarrow 0$ we get

$$
\frac{d I}{d t}+\alpha \beta t^{\beta-1} I(t)=-\lambda t^{-p} \quad \text { for } \quad 0 \leq t \leq T .
$$

Now solving equation (3.1) with boundary condition $I(T)=0$

$$
\begin{aligned}
& I(t)=\lambda\left(1-\alpha t^{\beta}\right)\left[\begin{array}{l}
\frac{1}{1-p}\left\{T^{1-p}-t^{1-\beta}\right\} \\
+\frac{\alpha}{1+\beta-p}\left\{T^{1+\beta-p}-t^{1+\beta-p}\right\}
\end{array}\right] \\
& \text { for } 0 \leq t \leq T \text {. }
\end{aligned}
$$

Using $I(0)=Q$, we get

$$
Q=\lambda\left[\frac{T^{1-p}}{1-p}+\frac{\alpha T^{1+\beta-p}}{1+\beta-p}\right] \text { for } 0 \leq t \leq T .
$$

The number of deteriorated units during one cycle time is given by.

$$
D(T)=Q-\int_{0}^{T} R(t) d t=\frac{\lambda \alpha T^{1+\beta-p}}{1+\beta-p} .
$$

The average inventory during the time interval $[0, T]$ is given by,

$$
\begin{aligned}
& I_{1}(T)=\int_{0}^{T} I(t) d t \\
& =\lambda\left[\frac{T^{2-p}}{2-p}+\frac{\alpha \beta}{(1+\beta)(2+\beta-p)} T^{2+\beta-p}\right]
\end{aligned}
$$

The total cost per unit time $C(T)$ comprises of following costs:

1. Purchasing cost per cycle

$$
p_{C} I(0)=\lambda p_{C}\left[\frac{T^{1-p}}{1-p}+\frac{\alpha T^{1+\beta-p}}{1+\beta-p}\right]
$$

2. Holding cost per cycle

$$
h_{c} \int_{0}^{T} I(t) d t=\lambda h_{c}\left[\frac{T^{2-p}}{2-p}+\frac{\alpha \beta}{(1+\beta)(2+\beta-p)} T^{2+\beta-p}\right]
$$

3. Deterioration cost per cycle

$$
d_{c} D(T)=\frac{d_{c} \lambda \alpha T^{1+\beta-p}}{1+\beta-p}
$$

4. Ordering cost

$$
O C=A
$$

5. Salvage value of deteriorated items

$$
S V=\frac{\gamma p_{c} \lambda \alpha T^{1+\beta-p}}{1+\beta-p}
$$

The average total cost per unit time of the model will be

$$
\begin{aligned}
C(T) & =\frac{1}{T}\left[\lambda p_{C}\left[\frac{T^{1-p}}{1-p}+\frac{\alpha T^{1+\beta-p}}{1+\beta-p}\right]\right. \\
& +\lambda h_{c}\left[\frac{T^{2-p}}{2-p}+\frac{\alpha \beta}{(1+\beta)(2+\beta-p)} T^{2+\beta-p}\right] \\
& \left.+\frac{d_{c} \lambda \alpha T^{1+\beta-p}}{1+\beta-p}+A-\frac{\gamma p_{c} \lambda \alpha T^{1+\beta-p}}{1+\beta-p}\right]
\end{aligned}
$$

The necessary condition for $C(T)$ to be minimum is $\frac{\partial C(T)}{\partial T}=0$ and solving it for $T$ by using a suitable software. For the $T$ obtained, $C(T)$ is minimum only if

$$
\begin{aligned}
& \frac{\partial^{2} C(T)}{\partial T^{2}}=\frac{p_{C} \lambda p(p+1) T^{-p-2}}{1-p} \\
& -\frac{h_{C} \lambda p(1-p) T^{-p-1}}{2-p} \\
& +\frac{h_{C} \lambda \alpha \beta(1+\beta-p)(\beta-p) T^{\beta-p-1}}{(1+\beta)(2+\beta-p)}+\frac{2 A}{T^{3}} \\
& +\frac{\left\{d_{C}-(\gamma-1) p_{C}\right\} \lambda \alpha(\beta-p)(\beta-p-1) T^{\beta-p-2}}{1+\beta-p}>0 \\
& \text { for all } T>0 .
\end{aligned}
$$

\section{Numerical example}

Following example is considered to illustrate the preceding theory.

\section{Example}

The values of the parameters are considered as follows: $\alpha=0.1, \beta=2, \gamma=0.2, A=300, \lambda=3000, p=0.2$, 
$d_{c}=\$ 8 /$ unit, $h_{c}=\$ 3 /$ unit, $p_{c}=\$ 20 /$ unit According to equation (3.11), we obtain the optimal $T=1.3663$.

\begin{tabular}{|c|c|c|c|c|}
\hline$\alpha$ & $T$ & $Q$ & $D(T)$ & $C(T)$ \\
\hline 0.1 & 1.36632 & 16222.95632 & 256.7497 & 81987.35856 \\
\hline
\end{tabular}

\section{Conclusion}

Here we have derived an inventory model for deteriorating items. In particular deterioration is considered to be of Weibull type. In this model, shortages are not allowed. An optimal replenishment policy is derived with minimization of average total cost under the influence of time varying demand. The result is illustrated through numerical example.

\section{References}

[1] Bahari - Kashani, H.: "Replenishment schedule for deteriorating items with time-proportional demand", Journal of the Operational Research Society, Vol. 40, 1989, pp. 75-81.

[2] Covert, R. B.; Philip, G. S.: "An EOQ model with Weibull distributed deterioration”, AIEEE Transactions, Vol. 5, 1973, pp. 323-326.

[3] Emmons, H.:"A replenishment model for radioactive nuclide generators”, Management Science, Vol. 14, 1968, 263-273.

[4] Ghare P.M. and Scharder G.P.: "A model for exponentially decaying inventory”, J. Ind. Eng., 14 (1963), 238-243.

[5] Goswami, A.; Chaudhuri, K. S.: "An EOQ model for deteriorating items with shortages and a linear trend in demand", Journal of the Operational Research Society, Vol. 42, 1991, pp. 1105-1110.

[6] Philip, G. C.: "A generalized EOQ model for items with Weibull distribution deterioration”, AIIE Transaction, Vol. 6, 1974, pp. 159-162.

[7] Tripathi, R.P. Inventory model with different demand rate and different holding cost, IJIEC, Volume 4, 2013, 437-446.

[8] Wagner, H.M.; Whitin T.M.:"'Dynamic version of the economic lot size model”, Management Science, Vol. 5(1), 1958, 89-96.

[9] Wee H.M.: "A deterministic lot-size inventory model for deteriorating items with shortages on a declining market”, Comp. Ops. Res., 22 (1995), 553-558. 